\title{
Use of MRI for Evaluation of Retained Uterine Fundus Mimicking a Pelvic Mass
}

\author{
Michael Reiter, DO*, James F. Wiedenhoefer, MD, Ryan Schwope, MD, Abigail Feathers, MD, \\ and Sarah Page, MD \\ From the Departments of Radiology (Drs. Reiter, Wiedenhoefer, and Schwope), and Obstetrics and Gynecology (Drs. Feathers and Page), Brooke Army \\ Medical Center, San Antonio, Texas.
}

\begin{abstract}
Increased use of less invasive hysterectomy techniques requires awareness of the unique potential complications they pose in patients with continued symptoms after surgery. Retained uterine fundus is rare after vaginal hysterectomy; only 2 other cases have been reported in the English literature. Magnetic resonance imaging (MRI) was not used preoperatively in either case. However, imaging evaluation, in particular with MRI because of its superior soft tissue resolution, can be helpful in suggesting the diagnosis. Herein is presented the case of a 40-year-old woman who had undergone vaginal hysterectomy several years previously, but was experiencing abdominal pain. MRI was performed, which revealed a supravesical mass. Visualization at MRI of intact round ligaments arising from the mass favored the diagnosis of retained uterine fundus and confirmed after surgical excision. Journal of Minimally Invasive Gynecology (2012) 19, 524-526 Published by Elsevier Inc. on behalf of AAGL.
\end{abstract}

Keywords: $\quad$ Retained uterine fundus; Vaginal hysterectomy; MRI

DISCUSS You can discuss this article with its authors and with other AAGL members at http://www.AAGL.org/jmig-19-4-12-00079

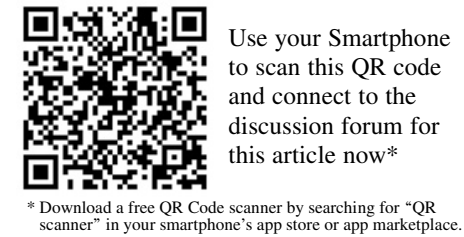

superior to the bladder (Fig. 1). The possibility of a parasitized leiomyoma was considered. The appendix was normal, excluding appendicitis, and no other acute abdominal findings were identified. Further patient questioning revealed that this episode of lower abdominal pain was superimposed on more chronic cyclic pelvic pain that had been occurring since the date of the hysterectomy.

At pelvic magnetic resonance imaging (MRI), the supravesical mass yielded a predominantly low signal on T2weighted images, and contained multiple hyperintense foci on T1-weighted sequences, which suggested hemorrhage. There was avid and homogeneous enhancement of the mass after administration of gadolinium contrast medium (Fig. 2). The left ovary was normal. There was a small hemorrhagic cyst in the right ovary. The uterus was not visualized, consistent with the history of hysterectomy. Linear structures, compatible with round ligaments, were visualized arising from this supravesical mass and extending toward the inguinal canals. In addition, a hyperintense curvilinear structure with a thin hypointense rim was noted centrally within this mass on thin-section T2-weighted images, which are

Submitted February 7, 2012. Accepted for publication March 21, 2012

Available at www.sciencedirect.com and www.jmig.org 


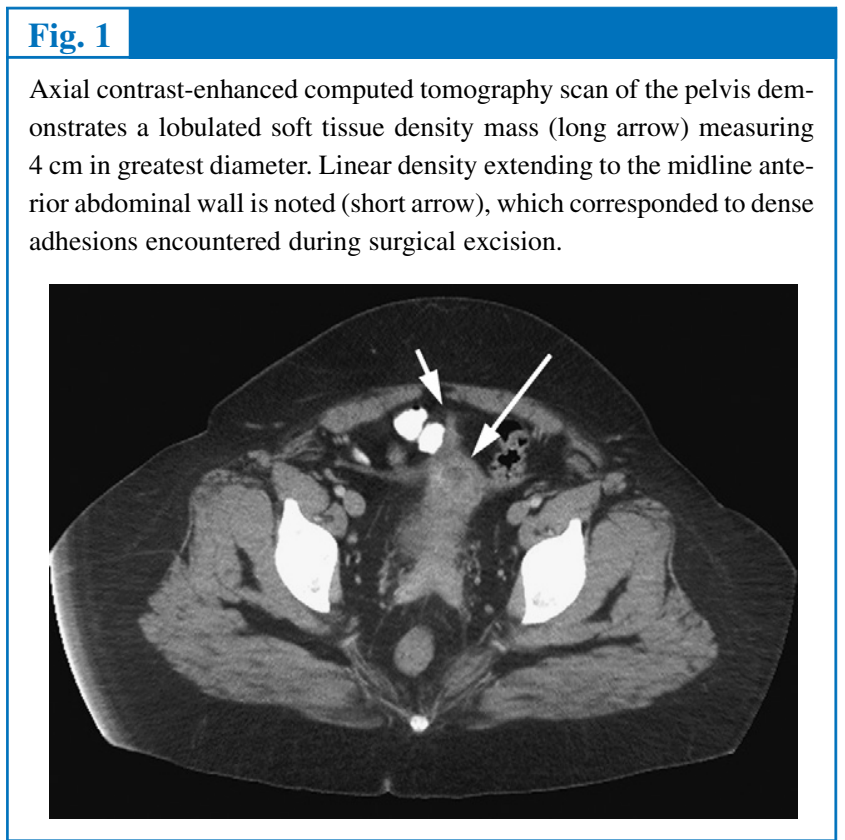

the signal characteristics of the endometrium and junctional zone, respectively (Fig. 3). The constellation of imaging findings favored a diagnosis of retained uterine fundus over a parasitized uterine leiomyoma.

The patient was taken to the operating room, and underwent laparoscopic evaluation of the presumed retained uterine fundus. Standard laparoscopic techniques were used for initial peritoneal entry. Laparoscopic evaluation revealed adhesive disease of the omentum and lower anterior abdominal wall, and thickened and scarred round ligaments attached to the retained uterine mass (Fig. 4). This dense adhesive dis-

\section{Fig. 2}

MRI of the pelvis. Coronal T1-weighted fat-saturated image obtained after intravenous contrast administration shows homogeneous enhancement of the mass (arrowhead). The round ligaments (arrows) are well delineated, arising from the mass and coursing toward the inguinal canals.

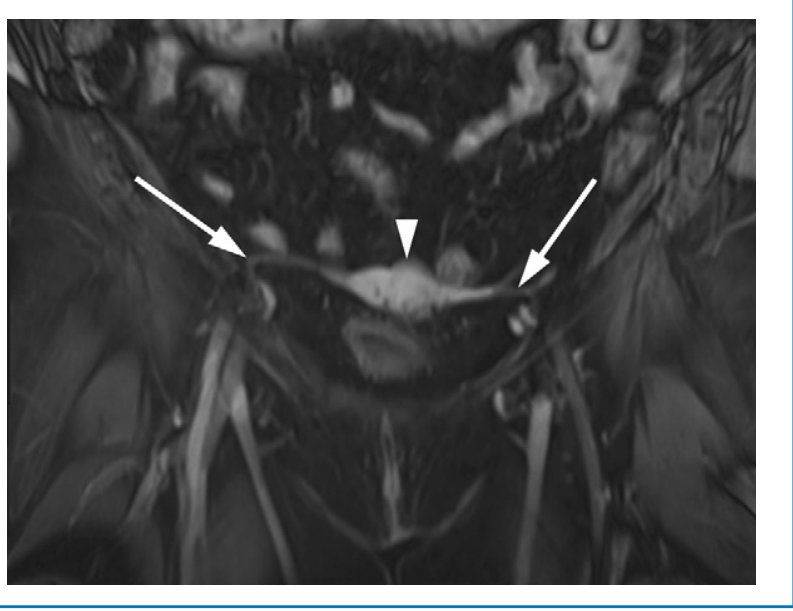

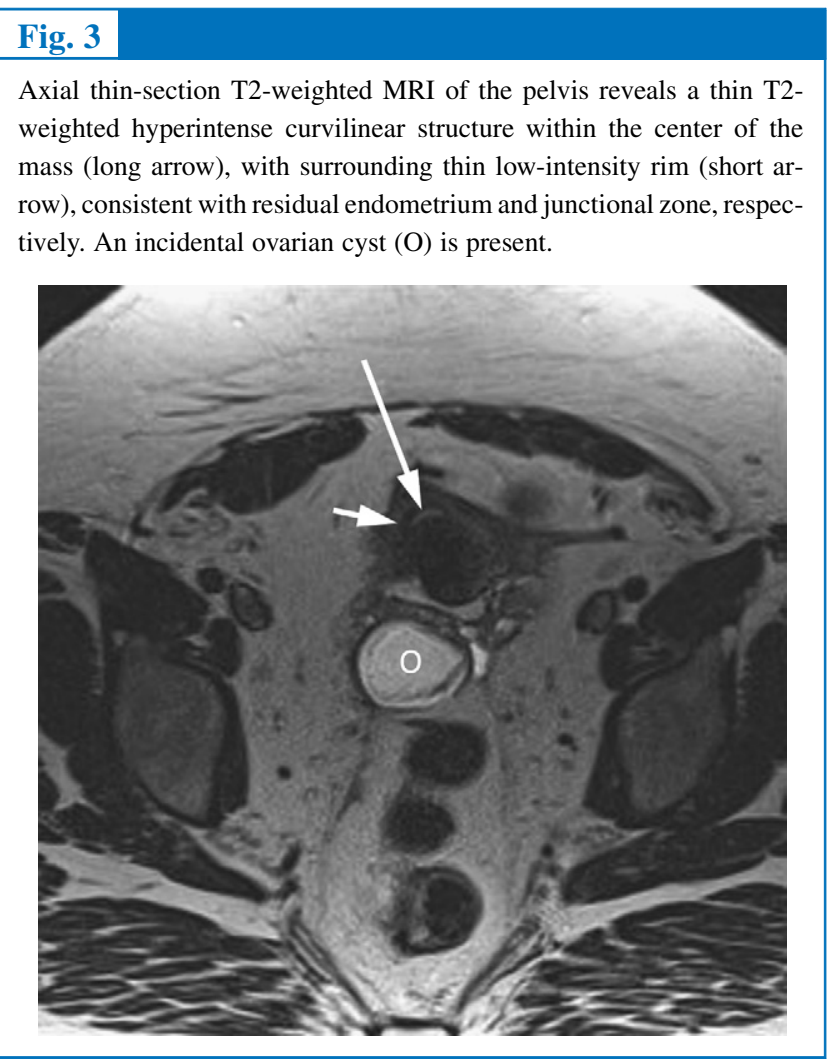

ease precluded reasonably safe laparoscopic surgical dissection, and the procedure was converted to laparotomy. Adhesiolysis was performed, and the retained uterine mass was subsequently removed via laparotomy. Bilateral oophorectomy was also performed. No intraoperative complications were encountered during laparotomy.

The surgical pathology report described a $3.5 \times 2.5-\mathrm{cm}$ mass with fragments of functional uterus and proliferative endometrium, and hemosiderin-laden macrophages compatible with previous bleeding. No hyperplasia or malignant lesion was identified.

\section{Fig. 4}

Laparoscopic image of the anterior cul-de-sac reveals the residual uterine fundus (F) adhered to the anterior abdominal wall by a dense fibrous band (D). The round ligaments are denoted by arrows.

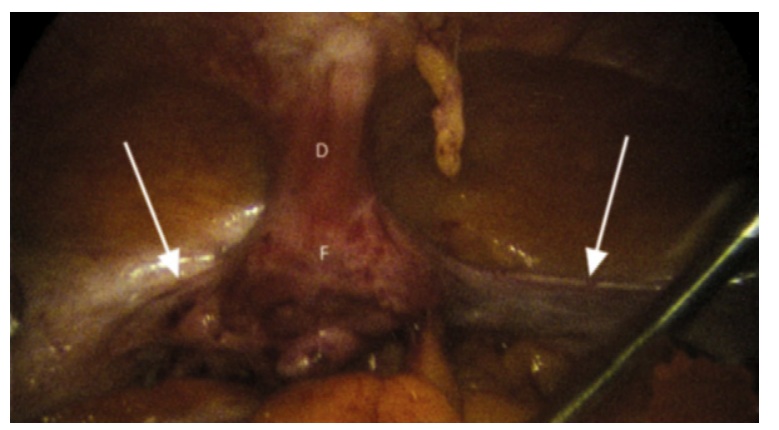


The patient did well postoperatively, and was discharged to home on postoperative day 2 .

\section{Discussion}

Second to cesarean delivery, hysterectomy is the most frequently performed surgical procedure in women of reproductive age. Approximately 600000 such procedures are performed each year. The 2009 Cochrane Review assessed the most beneficial surgical approach to hysterectomy to treat benign disease. Compared with abdominal hysterectomy, vaginal hysterectomy was more beneficial because it is substantially less invasive. If vaginal hysterectomy is not possible, laparoscopic hysterectomy can be considered to avoid the transabdominal approach [1]. One potential benefit to the laparoscopic approach compared with the vaginal approach is the ability to inspect the abdomen and pelvis for additional or retained masses. Contraindications to vaginal hysterectomy may include pelvic malignant disease, chronic pelvic inflammatory disease, and severe endometriosis. Several other relative contraindications have been suggested including previous pelvic surgery, large uterine size (gestational size $\geq 12$ weeks, or approximately 250-300 g), and the need for bilateral salpingo-oophorectomy. However, in experienced hands, these may not be considered contraindications [2].

Familiarity with less invasive hysterectomy techniques and their unique potential complications is important because they may become more frequently used. Morcellation, bivalving, and coring can be performed when the uterus is moderate or large in size, to facilitate its removal through either the laparoscopic port or the vagina. These techniques may also be performed in relatively immobile uteri regardless of size because they improve access to the broad ligaments and adnexae [3]. This, however, incurs the risk of leaving behind fragments of tissue if they are not identified and extracted at surgery. The entire abdominal cavity, therefore, must be meticulously inspected after morcellation is complete. In one series of 1405 patients undergoing laparoscopic subtotal hysterectomy, 8 women developed symptomatic adenomyomas within the pelvis several years after surgery, presumably due to growth of missed fragments of uterine tissue at morcellation [4]. In addition, parasitized uterus and cervical fragments within the pelvis have been reported in a 35-year-old woman after total laparoscopic hysterectomy [5].

Fragments of tissue inadvertently left behind after morcellation during hysterectomy have been well-documented. According to 1966-2011 PubMed literature searches of articles in the English language using the search terms "Hysterectomy," "Residual uterus," "Retained fundus," and "Retained uterus," this is only the third reported case of a retained portion of uterine fundus after vaginal hysterectomy, and the first in which MRI was performed [6]. As opposed to small residual fragments, a retained segment of functional uterine tissue incurs the risk of endometrial cancer, which would not be diagnosed until an advanced stage because the patient would not exhibit the classic symptom of vaginal bleeding. Because the operative notes from the initial surgery were not available for our patient, the specifics of the vaginal hysterectomy and whether morcellation was performed are unknown. After the initial vaginal hysterectomy, the surgeons told the patient they had a difficult time removing her uterus and that there was substantial bleeding. They also reported considering conversion to laparotomy intraoperatively, although they ultimately did not do so because they believed the procedure had been completed vaginally. Given the patient's history of cesarean deliveries, it is speculated that uterine adhesions complicated the attempted vaginal hysterectomy. This is supported by the presence of dense adhesive disease encountered at laparoscopy for planned excision of the retained uterine mass. However, it is uncertain whether the adhesive disease was present before the vaginal hysterectomy, a result of the vaginal hysterectomy, or a combination of the previous procedures. Of the previous 2 reported cases of fragmented tissue left behind after morcellation during hysterectomy, both women also had cesarean deliveries. Other similarities to our patient included morbid obesity, which is presumed to confound the clinical picture because the retained uterine fragment is not so easily palpable at physical examination.

While rare, retained uterine tissue after hysterectomy may be diagnosed initially via imaging. Appropriate knowledge of both uterine anatomy and its MRI appearance, including the round ligaments and expected uterine signal characteristics, is imperative. In cases of persistent pain after hysterectomy with a nonovarian pelvic soft tissue mass, MRI may be helpful in establishing the diagnosis of a partially retained uterus.

\section{References}

1. Nieboer TE, Johnson N, Lethaby A, et al. Surgical approach to hysterectomy for benign gynaecological disease. Cochrane Database Syst Rev. 2009;(3):CD003677.

2. Doucette RC, Sharp HT, Alder SC. Challenging generally accepted contraindications to vaginal hysterectomy. Am J Obstet Gynecol. 2001;184: 1386-1389.

3. Netto OF, Figueiredo EG, Figueiredo PG, et al. Vaginal removal of the benign nonprolapsed uterus: experience with 300 consecutive operations. Obstet Gynecol. 1999;94:348-351.

4. Donnez O, Squifflet J, Leconte I, et al. Posthysterectomy pelvic adenomyotic masses observed in 8 cases out of a series of 1405 laparoscopic subtotal hysterectomies. J Minim Invasive Gynecol. 2007;14:156-160.

5. LaCoursiere DY, Kennedy J, Hoffman CP. Retained fragments after total laparoscopic hysterectomy. J Minim Invasive Gynecol. 2005;12:67-69.

6. Sinha R, Lakhotia S, Sundaram M, et al. Retained uterine fundus after vaginal hysterectomy. J Minim Invasive Gynecol. 2010;17:94-96. 